\title{
Mathematical Analysis And Performance Evaluation Of The Cell-Free Mmimo Networks Based On Cognitive Relays
}

Mohamed Shalaby ( $\sim$ mhmd_shlpy@yahoo.com )

Higher Institute of Engineering and Technology in Tanta

Mohamed Shahein

Menoufia University

Mona Shokair

Menoufia University

\section{Research Article}

Keywords: Cell-Free mMIMO, MRC, Relays, Cognitive Radio, SE, EE.

Posted Date: February 14th, 2022

DOI: https://doi.org/10.21203/rs.3.rs-853356/v1

License: (c) (1) This work is licensed under a Creative Commons Attribution 4.0 International License.

Read Full License 


\title{
Mathematical Analysis and Performance Evaluation of the Cell-Free mMIMO Networks based on Cognitive Relays
}

\author{
Mohamed Shalaby ${ }^{(1)}$, Mohamed Shahein ${ }^{(2)}$, and Mona Shokair ${ }^{(2)}$ \\ (1) Higher Institute of Engineering and Technology in Tanta, Egypt \\ ${ }^{(2)}$ Faculty of Electronic Engineering, Menoufia University, Menouf, Egypt \\ Emails:mhmd_shlpy@yahoo.com,mosakr20202@gmail.com, and mona.sabry@el-eng.menofia.edu.eg
}

\begin{abstract}
The Cell-Free mMIMO networks can provide a satisfied performance for 5G networks and beyond even there are shadowed users or indoor ones. The performance of cell-Free mMIMO networks was mathematically analyzed and simulated. This work aims to apply the cognitive relays in cell-Free mMIMO networks. The cell-Free mMIMO system, based on cognitive relays, is mathematically analyzed and simulated. The proposed work depends on two axes. Firstly, the relays can improve the performance of cell-Free mMIMO networks especially at shadowed users and cell-edge ones. Secondly, the cognitive radio can mitigate the interference in a cell-Free mMIMO network. The Cell-Free mMIMO network, based on cognitive relays, is proposed. The system is mathematically analyzed and simulated. The spectral efficiency SE and signal to interference plus noise ratio SINR is deduced and given in a closed form formula. It can be concluded that the cognitive relays can improve; SINR, SE, and Energy Efficiency EE of a cell-Free mMIMO network.
\end{abstract}

Key words: Cell-Free mMIMO, MRC, Relays, Cognitive Radio, SE, EE.

\section{Introduction}

The realization tools of the 5G networks and beyond can include but not limited to; small cells, massive multiple input multiple output mMIMO, beamformers, and much more. The motivations towards having a small cell size are; high energy efficiency EE, high spectral efficiency SE, more resource reuse, improved signal to noise levels SNR at a receiver, and much more [1-4]. Other tools can include; MIMO and mMIMO systems. The implementation of a lot of antenna at a transmitter and a receiver can let the communication system have; diversity gain, beamforming gain, and spatial multiplexing gain [5-8]. The mMIMO is a powerful tool toward realization of the $6 \mathrm{G}$ networks. The massive number of implemented antennas at each transmitter and each receiver let the mMIMO system have an enhanced performance in comparable with a MIMO one. The mMIMO system can include at least 64 antennas at a transmitter or at a receiver. In general, both MIMO and mMIMO systems can provide a high uplink and downlink capacity.

The application of MIMO and mMIMO techniques can let a cellular system have a high performance; however there are a lot of weak points. These points can include; poor service for indoor users, shadowed and blockage users, cell-edges users, and much more. The users, which are at dead zones, should be served well as the $5 \mathrm{G}$ and the $6 \mathrm{G}$ networks promise to provide a ubiquitous coverage with a satisfied performance. To do this, a new technology called Cell-Free is launched. The Cell-Free mMIMO is an improved version of mMIMO technology wherein the massive number of centralized antennas at a base station is replaced by massive number distributed APs through the coverage area. In other words, the number of APs in a Cell-Free mMIMO is equal to the same number of antennas at a cellular mMIMO base station. The Cell-Free concept is shown in Figure 1 [9-11].

Since the Cell-Free mMIMO is an emerging technology for the $6 \mathrm{G}$ networks, there are a lot of researches that handled the topic in terms of mathematical analysis and simulation [9]. The cooperative communication is carried out among the APs in order to provide a reliable data transmission. The APs cooperation can be carried out by four levels which will be clarified in details in the following sections. Ref. [10] provided a mathematical analysis for a Cell-Free mMIMO in addition to application of low resolution analog to digital converters. These converters could improve the SE performance. Other trials were carried out in Ref. [11]. The ZeroForcing ZF as well as conjugate beam forming were applied in order to improve the uplink performance of a Cell-Free mMIMO system.

The basic concept of the Cell-Free mMIMO is the small size cells. A line of sight LOS operation should exist. However, the NLOS operation should be considered due to fading as well as Doppler spreader. The Cell-Free mMIMO system was analyzed when correlated fading channels were applied [12]. The authors tried to have a power efficient "green" Cell-Free mMIMO system by reduction of the consumed power in each antenna. As an extension of green Cell-Free mMIMO system, a scalable and energy efficient IOT system, with massive connectivity, was designed in Ref. [13]. 


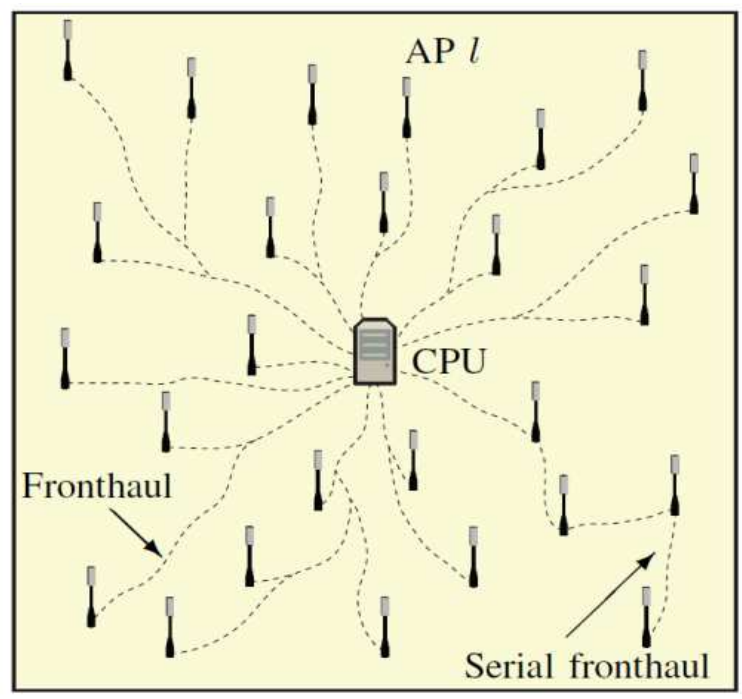

Figure 1: The Cell-Free mMIMO network [23].

Non orthogonal multiple access NOMA is a powerful tool in 5G networks and beyond. The NOMA can increase the SE of a cellular system. Application of NOMA in a Cell-Free mMIMO system promises to improve the SE performance [14]. The Cell-Free mMIMO, based NOMA with conjugate beamformer preceding, was analyzed and simulated. The authors held a comparison between NOMA based Cell-Free mMIMO system and OMA one in order to assure the performance of NOMA based Cell-Free mMIMO systems. In addition, the authors tried to drive a novel closed form SE formula that consider pilot contamination and other effects.

There are a lot of tools that can be applied in order to improve the SE and EE performance of a Cell-Free mMIMO system. In Ref. [15], a comprehensive SE optimization algorithm was applied. The optimization algorithm was based on power control algorithms with AP selection. The authors of Ref. [16] developed the work of Ref. [10] in order to introduce a mixed analog to digital converter ADC receiver architecture to a Cell-Free mMIMO system under Rician fading channels. The mixed ADC architecture permits some of receiver's antennas to be implemented with economical low resolution ADCs while the rests are equipped with high priced perfect ADCs.

The NOMA based Cell-Free mMIMO systems have a high SE performance. NOMA techniques were explained in a CellFree mMIMO [17]. It promises to increase SE as well as EE. On the other side, Ref. [18] concluded model and design of massive distributed APs system with an optical backhaul. Others concentrated on improving the EE of a Cell-Free mMIMO system. The authors of Ref. [19] analyzed a green Cell-Free mMIMO system in downlink operation. The half-duplex relays were added in a CellFree mMIMO system in order to maximize the SE [20]. Moreover, the system coverage was improved. The adaptive operation between small cells and Cell-Free mMIMO could maximize the SE performance [21]. The channel aging could affect the Cell-Free mMIMO system. This effect was analyzed and simulated [22].

The Cell-Free mMIMO network was mathematically modeled and simulated [23]. The Cell-Free mMIMO network can operate by applying four levels of cooperation mechanisms. The simulation was carried out through application of different number of APs and different number of antennas. Moreover, the SE of different users as well as the sum of spectral efficiency, after applying interference cancellation mechanisms, was simulated. Furthermore, in the previously mentioned work, the SE and EE based bit error rate BER performance of a Cell-Free mMIMO system were mathematically analyzed and they are given in a closed form formula.

Relays can be applied in a cellular system in order to provide a good coverage for; cell-edge users, indoor users, and shadowed users. They may be implemented in order to reduce the consumed power in a cellular system. Relays are powerful tools towards green communication systems. A cellular system, based on relays, can get benefits of cooperative communication process. For more clarifications, the receiver can combine the received signals from the original transmitter as well as the relays. This combination results in improvement in signal to noise ratio SNR. The increase in SNR results in reduction in bit error rate BER and increase in throughput. In brief, it is predicted that the Cell-Free mMIMO systems based on relays have a high SE and EE performance.

The cognitive radio is an emerging technology towards interference mitigation. The cognitive radio technology let two categories of users operate at the same time without considerable interference. The first category can operate at any time and these users are called primary users. On the other side, the other users can operate at vacant resources only and they are called secondary users. The cognitive radio is a powerful interference mitigation tool. 
The application of cognitive relays in a cellular system let the system have low interference and high EE. The low interference level can improve the signal to interference plus noise ratio SINR. In addition, the high EE levels let the cellular system to be a green one.

Our paper is organized as follows; Section 2 provides the mathematical analysis of the Cell-Free mMIMO network that is based on cognitive relays. Subsequently, the system is simulated and performance comparisons are held in Section 3. Finally, conclusions are given in Section 4.

\section{Cognitive Relay Based Cell-Free mMIMO}

Consider a cell-Free network model that has L distributed APs. Each AP has N antennas. The APs are connected over a centralized controller "cloud-edge processor". These APs can serve K users. Assume that $\mathrm{h}_{\mathrm{k}, \mathrm{l}}$ describes the channel between 1 AP and the $\mathrm{k}$ user. The coefficients of the channel vectors are assumed to have a Rayleigh distribution. The relays are randomly distributed inside a cell coverage area.

The Cell-Free mMIMO system, based on cognitive relays, is proposed assuming that the operation is in uplink. The UEs are transmitters whereas the APs are receivers. The APs can receive each data segment from UEs as well as cognitive relays. A cognitive relay can receive the UE signal and then, forward it to an AP. In fact, there are a lot of relaying strategies. However, this paper considers a traditional relay that follows decode and forward strategy.

\subsection{Proposed Cognitive Relay Based Cell-Free mMIMO Network}

In this section, the cognitive radio algorithm is applied between relays and APs in order to mitigate the interference between them. The cognitive radio can be applied according to the following steps;

- $\quad$ Each AP can distribute its resources among users.

- The relays should operate at vacant spectrum only that the APs do not operate on.

- By applying a cooperative sensing mechanism, the users can detect the spectrum holes that the APs do not operate on.

- The spectrum server "spectrum broker" can collect the vacant spectrum from the sensing information of all users. Then, these resources will be applied to relays.

- The relays can operate at the vacant resources only.

- The relays can operate by full duplex time division manner; in such a way that, both UE and relay can send to an AP in subsequent time slots. In other words, the AP can receive a UE data in a time slot and then it can receive its relayed version "from a relay" at the subsequent time slot.

- By application of cognitive radio concept between relays and APs, the SINR of a user can be reduced to be SNR only.

\subsection{Pilot Transmission}

Assume that there are mutually orthogonal pilot signals which are; $\varphi_{1}, \varphi_{2}, \varphi_{3}, \ldots, \varphi_{\tau p}$. These pilots are used for channel estimation during data transmission period. Since the pilots are used for control processes only, the total pilot length should not exceed $20 \%$ of total frame time in order to save the data rate.

The total available pilots can be divided into three group and they are;

- Group 1 is a summation of pilots that are applied to estimate the channel between UEs and cognitive relays.

- Group 2 is a summation of pilots that are applied to estimate the channel between cognitive relays and APs.

- Group 3 is a summation of pilots that are applied to estimate the channel between UEs and APs.

The total frame length is $\tau_{\mathrm{c}}$. The total frame length is divided into; pilot time and data time. The total pilot length is $\tau_{\mathrm{p}}$. Therefore, the data time can be $\left(\tau_{\mathrm{c}^{-}} \tau_{\mathrm{p}}\right)$. The total pilot length is divided into three equal parts. Each part has a length of $\tau_{\mathrm{p}} / 3$. Due to the limited number of pilots, the number of users may be higher than the number of pilots. A lot of users can operate on a pilot which results in a pilot contamination. In the proposed work, the pilots are divided into three groups. This division results in increased pilot contamination [23]. When the UEs transmit their pilots, the received pilot at a relay can be expressed as;

$$
\boldsymbol{Z}_{l S R}=\sum_{i=1}^{k} \sqrt{p_{i}} \boldsymbol{\beta}_{S R i l} \emptyset_{t_{i}}^{T}+\boldsymbol{N}_{L S R} \quad 0 \leq t \leq \frac{\mathcal{T}_{p}}{3}
$$

The pilots, that are applied to estimate the channel between a relay and an AP, can be mathematically expressed as; 


$$
\boldsymbol{Z}_{l R D}=\sum_{i=1}^{k} \sqrt{p_{i}} \boldsymbol{\beta}_{R D i l} \varnothing_{t_{i}}^{T}+\boldsymbol{N}_{L R D} \quad \frac{\mathcal{T}_{p}}{3}+1 \leq t \leq \frac{2 \mathcal{T}_{p}}{3}
$$

The pilots, that are applied to estimate the channel between a UE and an AP, can be mathematically expressed as;

$$
\boldsymbol{Z}_{l S D}=\sum_{i=1}^{k} \sqrt{p_{i}} \boldsymbol{\beta}_{S D i l} \varnothing_{t_{i}}^{T}+N_{L S D} \quad \frac{2 \mathcal{T}_{p}}{3}+1 \leq t \leq \mathcal{T}_{p}
$$

where $\boldsymbol{p}_{\mathbf{i}}$ is the transmit power of $\mathrm{i}^{\text {th }}$ user, $\boldsymbol{\beta}_{\mathrm{SR}}$ is the channel vector between a UE and a Relay, $\boldsymbol{\beta}_{\mathrm{RD}}$ is the channel vector between a Relay and an AP, $\boldsymbol{\beta}_{\mathrm{SD}}$ is the channel vector between a UE and an AP. The parameter of $\mathbf{N}_{\mathrm{LSR}}, \mathbf{N}_{\mathrm{LRD}}$, and $\mathbf{N}_{\mathrm{LSD}}$ is the noise signals. To estimate the channel parameters, the Relay and each AP should correlate the received pilot signals with a locally generated version of the pilot signal in order to determine the channel parameters.

The received UE pilot signal at a relay should be correlated with its replica in order to determine the channel parameters that exist between each UE and a relay. This correlation can be mathematically expressed as follow;

$$
Z_{t_{k} l}=\sum_{\boldsymbol{d}_{i=1}}^{k} \frac{\sqrt{\frac{p_{i}}{3}}}{\sqrt{\frac{T_{p}}{3}}} \boldsymbol{\beta}_{S R i l} \phi_{t_{i}}^{T} \emptyset_{t_{k}}^{*}+\frac{1}{\sqrt{\frac{p_{p}}{3}}} \boldsymbol{N}_{L S R} \emptyset_{t_{k}}^{*}=\sum_{i \in P_{k}} \sqrt{\frac{p_{i} \mathcal{T}_{p}}{3}} \boldsymbol{\beta}_{S R i l}+\boldsymbol{n}_{t_{k} l}
$$

where $\frac{\mathcal{T}_{p}}{3}$ is the time allowed for this pilot transmission. The MMSE is applied in order to estimate the channel parameter between a $\mathrm{UE}$ and a relay, $\hat{\beta}_{S R k l}$, can be given by;

$$
\hat{\beta}_{S R k l}=\sqrt{\frac{p_{k} \mathcal{T}_{p}}{3}} \boldsymbol{R}_{k l} \Psi_{t_{k} l}^{-1} z_{t_{k} l}
$$

where;

$$
\Psi_{t_{k} l}=\mathbb{E}\left\{\mathbf{z}_{t_{k} l} \boldsymbol{z}_{t_{k} l}^{H}\right\}=\sum_{i \in P_{k}} \frac{J_{p}}{3} p_{i} \boldsymbol{R}_{i l}+\boldsymbol{I}_{N}
$$

Eq. 6 gives an expression for the correlation matrix of the received signal at relays.

The first received pilot signal at an AP is that comes from relays. This plot can be applied in order to estimate the wireless channel between a relay and an AP. The received pilot is correlated with a locally generated replica. This correlation can be mathematically expressed as follow;

$$
Z_{t_{k} l}=\sum_{i=1}^{k} \frac{\sqrt{p_{i}}}{\sqrt{\frac{\mathcal{T}_{p}}{3}}} \boldsymbol{\beta}_{R D i l} \emptyset_{t_{i}}^{T} \emptyset_{t_{k}}^{*}+\frac{1}{\sqrt{\frac{\mathcal{T}_{p}}{3}}} \boldsymbol{N}_{L R D} \emptyset_{t_{k}}^{*}=\sum_{i \in P_{k}} \sqrt{\frac{p_{i} \mathcal{T}_{p}}{3}} \boldsymbol{\beta}_{R D i l}+\boldsymbol{n}_{t_{k} l}
$$

where $\frac{\mathcal{T}_{p}}{3}$ is the time allowed for pilot transmission. By using the MMSE to estimate the channel parameter between a relay and an AP, $\hat{\beta}_{R D k l}$, can be given by;

$$
\hat{\beta}_{R D k l}=\sqrt{\frac{p_{k} \mathcal{T}_{p}}{3}} \boldsymbol{R}_{k l} \Psi_{t_{k} l}^{-1} z_{t_{k}} l
$$

where;

$$
\Psi_{t_{k} l}=\mathbb{E}\left\{\boldsymbol{z}_{t_{k} l} \boldsymbol{z}_{t_{k} l}^{H}\right\}=\sum_{i \in P_{k}} \frac{J_{p}}{3} p_{i} \boldsymbol{R}_{i l}+\boldsymbol{I}_{N}
$$

Eq. 9 gives an expression for the correlation matrix of the first received pilot signal at an AP.

The second received pilot signal at an AP is that comes from UEs. This plot can be applied in order to estimate the wireless channel between a UE and an AP. The received pilot is correlated with a locally generated replica. This correlation can be mathematically expressed as follow; 


$$
Z_{t_{k} l}=\sum_{i=1}^{k} \frac{\sqrt{p_{i}}}{\sqrt{\frac{\mathcal{T}_{p}}{3}}} \boldsymbol{\beta}_{S D i l} \emptyset_{t_{i}}^{T} \emptyset_{t_{k}}^{*}+\frac{1}{\sqrt{\frac{\mathcal{T}_{p}}{3}}} \boldsymbol{N}_{L S D} \emptyset_{t_{k}}^{*}=\sum_{i \in P_{k}} \sqrt{\frac{p_{i} \mathcal{T}_{p}}{3}} \boldsymbol{\beta}_{S D i l}+\boldsymbol{n}_{t_{k} l}
$$

where $\frac{\mathcal{J}_{p}}{3}$ is the time allowed for pilot transmission By using the MMSE to estimate the channel parameter between a UE and an AP, $\hat{\beta}_{S D k l}$, can be given by;

$$
\hat{\beta}_{S D k l}=\sqrt{\frac{p_{k} \mathcal{J}_{p}}{3}} \boldsymbol{R}_{k l} \Psi_{t_{k} l}^{-1} z_{t_{k} l}
$$

where;

$$
\Psi_{t_{k} l}=\mathbb{E}\left\{\mathbf{z}_{t_{k} l} \mathbf{z}_{t_{k} l}^{H}\right\}=\sum_{i \in P_{k}} \frac{J_{p}}{3} p_{i} \boldsymbol{R}_{i l}+\boldsymbol{I}_{N}
$$

Eq. 12 gives an expression for the correlation matrix of the second received pilot signal at an AP.

\subsection{Data Transmission}

During the uplink data transmission, the total received complex base band signals from UE and cognitive Relay can be mathematically modeled as;

$$
y=\sum_{i=1}^{k} \boldsymbol{\beta}_{S D i l} s_{i}+\sum_{i=1}^{k} \boldsymbol{\beta}_{R D i l} s_{R}+n
$$

where $y$ is the received signal, $\boldsymbol{S}_{i}$ is the transmitted signal from UE, while $\boldsymbol{S}_{R}$ is the transmitted signal from relay, $n$ is the channel noise, and $\boldsymbol{\beta}_{\mathrm{RD}}$ is the channel vector between a cognitive relay and an AP, $\boldsymbol{\beta}_{\mathrm{SD}}$ is the channel vector between a UE and an AP that can include the path loss and shadowing.

\subsubsection{Cooperation among the APs}

The basic concept depends on the idea of the Cell-Free mMIMO network based on cognitive relays. The coverage area is totally covered by distributed APs, in such a way that, each user equipment can have a service from the nearest AP as well as the nearest cognitive relay. Combination between AP signal and the cognitive relay one can improve the SNR and this let the system be a power efficient one. The cooperation among the several APs and cognitive relays can be carried out by four levels which are stated below.

\section{- Fully Centralized APs "Level 4"}

During this cooperation mechanism, the APs can provide service as well as relays. They can receive both pilot signals and the data and then send them directly to a central processing unit. The centralized controller can carry out the signal processing algorithms on the data which are received from all APs. The centralized controller can have processing capability more than an AP. The received signal, $\mathrm{Y}$, can be expressed as a function of the transmitted signal, $\mathrm{S}_{\mathrm{i}}$ and $\mathrm{S}_{\mathrm{R}}$ as follow

$$
y=\sum_{i=1}^{k} \boldsymbol{\beta}_{S D i l} s_{i}+\sum_{i=1}^{k} \boldsymbol{\beta}_{R D i l} s_{R}+n
$$

where $n$ is the noise signal and $\boldsymbol{\beta}_{\mathrm{RD}}$ is the channel vector between a cognitive relay and an $\mathrm{AP}$, and $\boldsymbol{\beta}_{\mathrm{SD}}$ is the channel vector between a UE and an AP that can include the path loss and shadowing. The signal to interference and noise ratio, SINR, and the spectral efficiency, $S E$, and can be calculated by the following relations;

$$
\begin{array}{r}
\text { (15) } \operatorname{SINR}_{k}^{(4)}=\frac{p_{k}\left|V_{k}^{H} \widehat{\boldsymbol{\beta}}_{S D K}\right|^{2}+p_{k}\left|V_{k}^{H} \widehat{\boldsymbol{\beta}}_{R D K}\right|^{2}}{V_{k}^{H} \boldsymbol{\sigma}^{2} \boldsymbol{I}_{L N} V_{k}} \\
S E_{K}^{(4)}=\frac{1}{2}\left(1-\frac{T_{p}}{T_{c}}\right) \mathbb{E}\left\{\log _{2}\left(1+\beta_{S D} \operatorname{SINR}^{(4)}+\min \left(\beta_{S R} \times \beta_{R D}\right) \times \operatorname{SINR}^{(4)}\right)\right\}
\end{array}
$$

With the help of Ref. [23], the energy efficiency, $E E$, can be expressed as;

$$
E E=B W \frac{S E}{P_{c}+P_{T}}
$$


where $B W$ is the bandwidth, $P_{C}$ is the power consumed in the circuits, and $P_{T}$ is the transmitted power. The transmission power, during the uplink is the mobile equipment power. The $E E$ can be calculated per unity $B W$ value.

\section{- Level 3}

During this level of cooperation, each $A P$ can detect the pilot signal in order to perform the channel estimates. Then, the estimated channel parameters associated with the received data signals are sent to the centralized controller. The central controller can detect the data signal based on the channel estimates. This level can largely ease the complexity of computation at the central controller. The signal to interference plus noise ratio, SINR, and the $S E$ can be expressed as;

$$
\begin{gathered}
(18) \operatorname{SINR}_{k}^{(3)}=\frac{\left.p_{k}\left|a_{k}^{H} \mathbb{E}\left\{g_{k k}\right\}\right|^{2}\left|S+p_{k}\right| a_{k}^{H} \mathbb{E}\left\{g_{k k}\right\}\right|^{2} \mid R}{\sigma^{2} a_{k}^{H} D_{k} a_{k}} \\
\left.S E_{K}^{(3)}=\frac{1}{2}\left(1-\frac{\mathcal{T}_{p}}{\mathcal{T}_{c}}\right) \log _{2}\left(1+\boldsymbol{\beta}_{S D} \operatorname{SINR} R_{k}^{(3)}+\min \left(\boldsymbol{\beta}_{S R}, \boldsymbol{\beta}_{R D}\right)\right) \operatorname{SINR}_{k}^{(3)}\right)
\end{gathered}
$$

\section{- Level 2}

In this cooperation level, the centralized controller can receive the estimated channel parameters and the received data. Then, it can detect the received data based on the average of the channel estimates. The average of the channel estimates can ease the computation complexity at the centralized controller. The spectral efficiency, SE, and the signal to interference plus noise ratio, SINR, can be calculated by the following relations;

$$
\begin{array}{r}
S E_{K}^{(2)}=\frac{1}{2}\left(1-\frac{\mathcal{T}_{p}}{\mathcal{T}_{c}}\right) \log _{2}\left(1+\boldsymbol{\beta}_{S D} \operatorname{SINR}_{k}^{(2)}+\min \left(\boldsymbol{\beta}_{S R}, \boldsymbol{\beta}_{R D}\right) \operatorname{SINR}_{k}^{(2)}\right) \\
(21) \operatorname{SIN} R_{k}^{(2)}=\frac{p_{k}\left|\sum_{i=1}^{k} \mathbb{E}\left\{V_{k l}^{H} \boldsymbol{\beta}_{S D l}\right\}\right|^{2}+p_{k}\left|\sum_{i=1}^{k} \mathbb{E}\left\{V_{k l}^{H} \boldsymbol{\beta}_{R D l}\right\}\right|^{2}}{\sigma^{2} \sum_{l=1}^{k} \mathbb{E}\left\{\left\|V_{k l}\right\|^{2}\right\}}
\end{array}
$$

The energy efficiency, $E E$, can be calculated by applying a unity $B W$ value in Eq. 17 .

\section{- Fully Distributed Level 1}

In this cooperation level, the APs can detect both pilot signal and data signal. The processing functions are carried out at the APs. Therefore, the centralized controller is free from pilot or data detection. The centralized controller, in this case, can provide cooperation among the APS only when it receives the detected pilots and estimated data for all users. The spectral efficiency, $S E$, and the signal to interference plus noise ratio, SINR, can be calculated by the following relations;

$$
S E_{K}^{(1)}=\frac{1}{2}\left(1-\frac{\mathcal{T}_{p}}{\mathcal{T}_{c}}\right) \max _{l \in\{1, \ldots ., L\}} \mathbb{E}\left\{\log _{2}\left(1+\boldsymbol{\beta}_{S D} \operatorname{SINR}(1)+\min \left(\boldsymbol{\beta}_{S R}, \boldsymbol{\beta}_{R D}\right) \operatorname{SIN} R_{k}^{(1)}\right)\right\}
$$

$$
(23) S I N R_{k}^{(1)}=\frac{p_{k}\left|V_{k l}^{H} \widehat{\boldsymbol{\beta}}_{S D K l}\right|^{2}+p_{k}\left|V_{k l}^{H} \widehat{\boldsymbol{\beta}}_{R D K l}\right|^{2}}{V_{k l}^{H} \boldsymbol{\sigma}^{2} \boldsymbol{I}_{N} V_{k l}}
$$

The EE can be calculated as in Eq. 17.

\section{Simulation Results}

In this section, the Cell-Free mMIMO network, based on relays, is simulated. The simulation parameters are concluded in Table 1 . The cell radius is $20 \mathrm{~m}$. In addition, the cognitive relays are randomly distributed inside the coverage area. However, the best place of a relay deployment is at the half distance between a transmitter and a receiver. The channel propagation and fading models are applied according to 3GPP standard [23]. The path loss models are given in Eq. 24 and Eq. 25. These models are applied in forementioned work [23].

$$
\begin{gathered}
\beta_{k l}[d B]=-30.5-36.7 \log _{10}\left(\frac{d_{k l}}{1 m}\right)+F_{k l} \\
\mathbb{E}\left\{F_{k l} F_{i j}\right\}= \begin{cases}4^{2} 2^{-\delta_{k i} / 9 m} & l=j \\
0 & l \neq j\end{cases}
\end{gathered}
$$


In this section, the Cell-Free mMIMO network, based on cognitive relays, is simulated. The simulation parameters are concluded in Table 1 . The cell radius is $20 \mathrm{~m}$. In addition, the cognitive relays are randomly distributed inside the coverage area. However, the best place of a cognitive relay deployment is at the half distance between a transmitter and a receiver. The channel propagation and fading models are applied according to 3GPP standard [23]. The path loss models are given in Eq. 24 and Eq. 25. These models are applied in fore-mentioned work [23].

Figure 2 shows the SE performance of a cell-Free mMIMO system without and with cognitive relays when the MRC is applied. It can be observed that the cognitive relay deployment can enhance the performance of a cell-Free mMIMO network. The four levels of cooperation can have a better performance when the relays exist.

Figure 3 displays the EE performance of a cell-Free mMIMO system without and with cognitive relays when the MRC is applied. It can be observed that the cognitive relay deployment can enhance the EE performance of a cell-Free mMIMO network. The four levels of cooperation can have a better performance when the relays exist.

Table 1. The simulation parameters.

\begin{tabular}{|c|c|c|}
\hline \multicolumn{2}{|r|}{ Parameter } & Value \\
\hline \multicolumn{2}{|c|}{ Number of cellular base stations } & 4 \\
\hline \multicolumn{2}{|c|}{ Number of antennas per each base station } & 100 \\
\hline \multicolumn{2}{|r|}{ Area } & $1 \times 1 \mathrm{~km}$ \\
\hline \multicolumn{2}{|r|}{ Fading } & Rayleigh Fading \\
\hline \multirow{3}{*}{ Shadowing } & Standard Deviation & $4 \mathrm{~dB}$ \\
\hline & Correlation Distance among UEs & $0.5 \mathrm{~m}$ \\
\hline & Decorrelation distance & $9 \mathrm{~m}$ \\
\hline \multicolumn{2}{|r|}{ Noise Figure } & $9 \mathrm{~dB}$ \\
\hline \multicolumn{2}{|r|}{ Bandwidth } & $20 \mathrm{MHz}$ \\
\hline \multicolumn{2}{|r|}{ Antenna Spacing } & 0.5 \\
\hline \multicolumn{2}{|c|}{ UE Transmission power } & $20 \mathrm{dBm}$ \\
\hline \multicolumn{2}{|c|}{ Number of UEs } & 40 \\
\hline \multicolumn{2}{|r|}{$P_{T}$} & $100 \mathrm{mWatt}$ \\
\hline \multicolumn{2}{|r|}{$P_{C}$} & 0.1 Watt \\
\hline
\end{tabular}

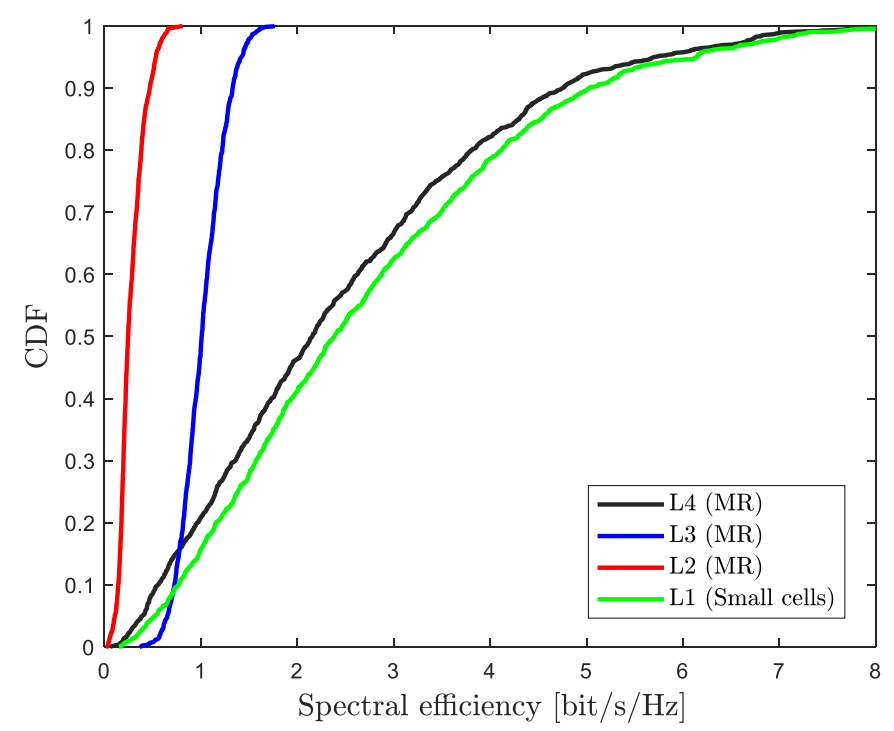

(a) Without cognitive relays

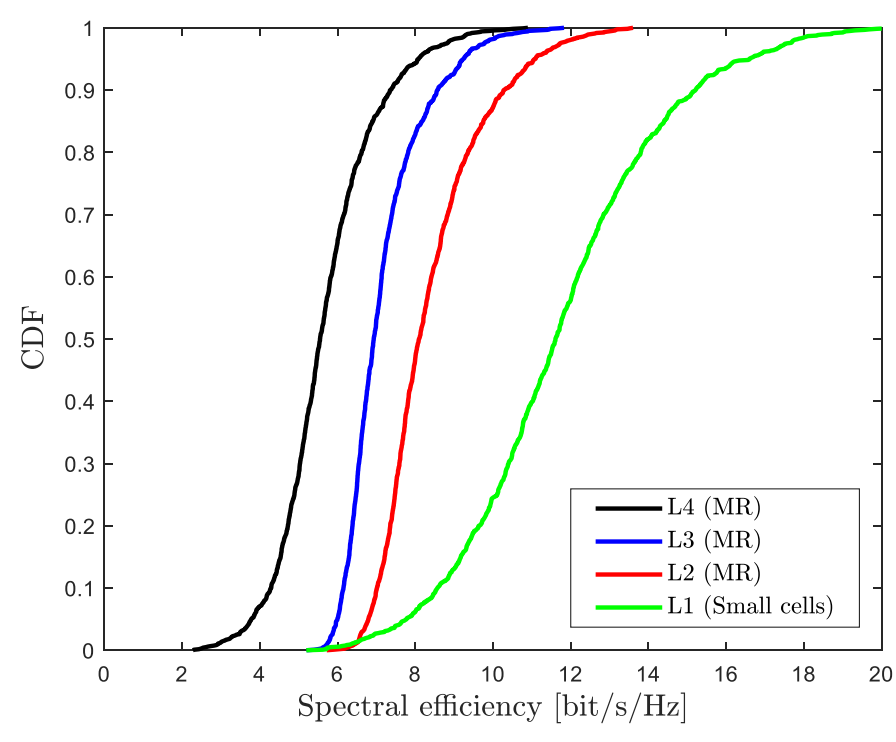

(b) With cognitive relays

Figure 2: The spectral efficiency performance of a Cell-Free mMIMO system based on cognitive relays. 


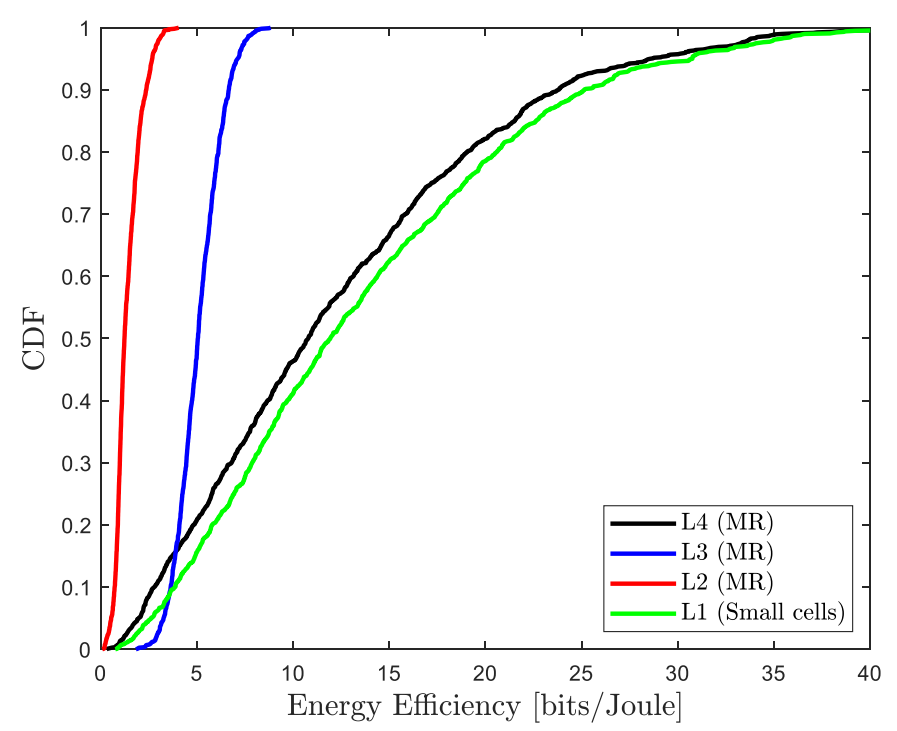

(a) Without cognitive relays

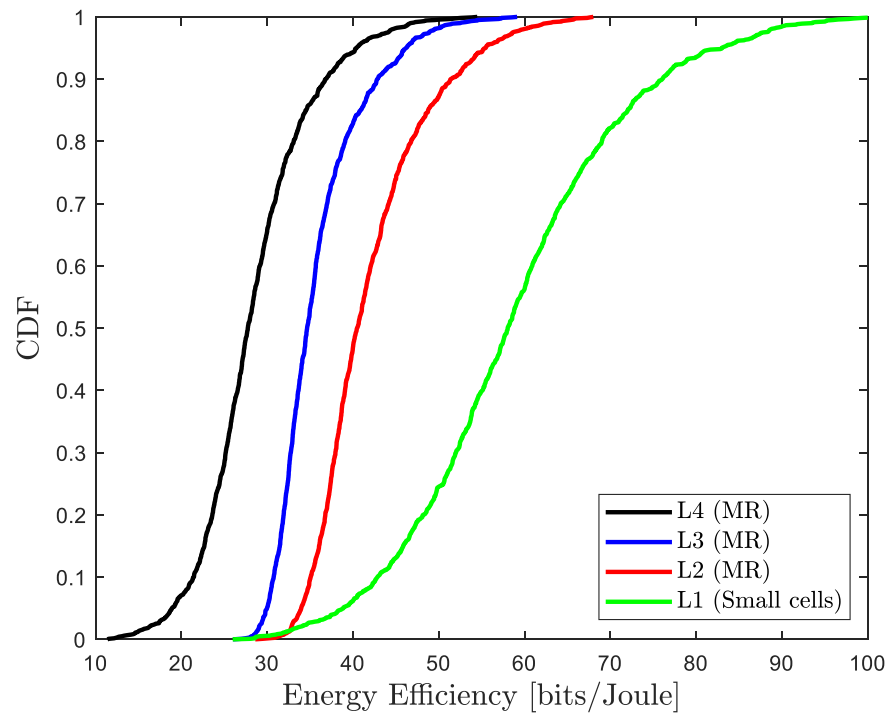

(b) With cognitive relays

Figure 3: The energy efficiency performance of a Cell-Free mMIMO system based on cognitive relays.

\section{Declarations}

The datasets generated during and/or analyzed during the current study are available from the corresponding author on reasonable request.

Funding source of this work is supported by the faculty of Electronic Engineering, Menoufia University, Menouf, Egypt.

There is no conflict between this work and other published work.

The Mathlab code is available on reasonable request.

\section{References}

1. D. Muirhead, M. A. Imran and K. Arshad, "A Survey of the Challenges, Opportunities and Use of Multiple Antennas in Current and Future 5G Small Cell Base Stations," in IEEE Access, vol. 4, pp. 2952-2964, 2016, doi: 10.1109/ACCESS.2016.2569483.

2. Y. Yang, B. Bai and W. Chen, "Spectrum Reuse Ratio in 5G Cellular Networks: A Matrix Graph Approach," in IEEE Transactions on Mobile Computing, vol. 16, no. 12, pp. 3541-3553, 1 Dec. 2017, doi: 10.1109/TMC.2017.2696005.

3. A. Taufique, M. Jaber, A. Imran, Z. Dawy and E. Yacoub, "Planning Wireless Cellular Networks of Future: Outlook, Challenges and Opportunities," in IEEE Access, vol. 5, pp. 4821-4845, 2017, doi: 10.1109/ACCESS.2017.2680318.

4. F. Song et al., "Probabilistic Caching for Small-Cell Networks With Terrestrial and Aerial Users," in IEEE Transactions on Vehicular Technology, vol. 68, no. 9, pp. 9162-9177, Sept. 2019, doi: 10.1109/TVT.2019.2929839.

5. Y. Xin, D. Wang, J. Li, H. Zhu, J. Wang and X. You, "Area Spectral Efficiency and Area Energy Efficiency of Massive MIMO Cellular Systems," in IEEE Transactions on Vehicular Technology, vol. 65, no. 5, pp. 3243-3254, May 2016, doi: 10.1109/TVT.2015.2436896.

6. Shojaeifard, K. Wong, M. Di Renzo, G. Zheng, K. A. Hamdi and J. Tang, "Massive MIMO-Enabled Full-Duplex Cellular Networks," in IEEE Transactions on Communications, vol. 65, no. 11, pp. 4734-4750, Nov. 2017, doi: 10.1109/TCOMM.2017.2731768.

7. Y. Han, B. D. Rao and J. Lee, "Massive Uncoordinated Access With Massive MIMO: A Dictionary Learning Approach," in IEEE Transactions on Wireless Communications, vol. 19, no. 2, pp. 1320-1332, Feb. 2020, doi: 10.1109/TWC.2019.2952843.

8. L. You et al., "Pilot Reuse for Vehicle-to-Vehicle Underlay Massive MIMO Transmission," in IEEE Transactions on Vehicular Technology, vol. 69, no. 5, pp. 5693-5697, May 2020, doi: 10.1109/TVT.2020.2982013.

9. E. Björnson and L. Sanguinetti, "Making Cell-Free Massive MIMO Competitive With MMSE Processing and Centralized Implementation," in IEEE Transactions on Wireless Communications, vol. 19, no. 1, pp. 77-90, Jan. 2020, doi: 10.1109/TWC.2019.2941478.

10. Y. Zhang, M. Zhou, X. Qiao, H. Cao and L. Yang, "On the Performance of Cell-Free Massive MIMO With Low-Resolution ADCs," in IEEE Access, vol. 7, pp. 117968-117977, 2019, doi: 10.1109/ACCESS.2019.2937094.

11. Y. Zhang, H. Cao, M. Zhou and L. Yang, "Cell-free massive MIMO: Zero forcing and conjugate beamforming receivers," in Journal of Communications and Networks, vol. 21, no. 6, pp. 529-538, Dec. 2019, doi: 10.1109/JCN.2019.000053.

12. J. Qiu, K. Xu, X. Xia, Z. Shen and W. Xie, "Downlink Power Optimization for Cell-Free Massive MIMO Over Spatially Correlated Rayleigh Fading Channels," in IEEE Access, vol. 8, pp. 56214-56227, 2020, doi: 10.1109/ACCESS.2020.2981967. 
13. S. Chen, J. Zhang, Y. Jin and B. Ai, "Wireless powered IoE for 6G: Massive access meets scalable cell-free massive MIMO," in China Communications, vol. 17, no. 12, pp. 92-109, Dec. 2020, doi: 10.23919/JCC.2020.12.007.

14. Y. Zhang, H. Cao, M. Zhou and L. Yang, "Non-orthogonal multiple access in cell-free massive MIMO networks," in China Communications, vol. 17, no. 8, pp. 81-94, Aug. 2020, doi: 10.23919/JCC.2020.08.007.

15. H. V. Nguyen et al., "On the Spectral and Energy Efficiencies of Full-Duplex Cell-Free Massive MIMO," in IEEE Journal on Selected Areas in Communications, vol. 38, no. 8, pp. 1698-1718, Aug. 2020, doi: 10.1109/JSAC.2020.3000810.

16. Y. Zhang, M. Zhou, H. Cao, L. Yang and H. Zhu, "On the Performance of Cell-Free Massive MIMO With Mixed-ADC Under Rician Fading Channels," in IEEE Communications Letters, vol. 24, no. 1, pp. 43-47, Jan. 2020, doi: 10.1109/LCOMM.2019.2947462.

17. O. Maraqa, A. S. Rajasekaran, S. Al-Ahmadi, H. Yanikomeroglu and S. M. Sait, "A Survey of Rate-Optimal Power Domain NOMA With Enabling Technologies of Future Wireless Networks," in IEEE Communications Surveys \& Tutorials, vol. 22, no. 4, pp. 2192-2235, Fourthquarter 2020, doi: 10.1109/COMST.2020.3013514.

18. L. Yu, J. Wu, A. Zhou, E. G. Larsson and P. Fan, "Massively Distributed Antenna Systems With Nonideal Optical Fiber Fronthauls: A Promising Technology for 6G Wireless Communication Systems," in IEEE Vehicular Technology Magazine, vol. 15, no. 4, pp. 43-51, Dec. 2020, doi: 10.1109/MVT.2020.3018100.

19. A. Papazafeiropoulos, H. Q. Ngo, P. Kourtessis, S. Chatzinotas and J. M. Senior, "Towards Optimal Energy Efficiency in Cell-Free Massive MIMO Systems," in IEEE Transactions on Green Communications and Networking, vol. 5, no. 2, pp. 816-831, June 2021, doi: 10.1109/TGCN.2021.3059206.

20. A. K. Papazafeiropoulos, P. Kourtessis, S. Chatzinotas and J. M. Senior, "Multipair Two-Way DF Relaying With Cell-Free Massive MIMO," in IEEE Open Journal of the Communications Society, vol. 2, pp. 423-438, 2021, doi: 10.1109/OJCOMS.2021.3060661.

21. S. Elhoushy and W. Hamouda, "Towards High Data Rates in Dynamic Environments Using Hybrid Cell-Free Massive MIMO/Small-Cell System," in IEEE Wireless Communications Letters, vol. 10, no. 2, pp. 201-205, Feb. 2021, doi: 10.1109/LWC.2020.3021026.

22. R. Chopra, C. R. Murthy and A. K. Papazafeiropoulos, "Uplink Performance Analysis of Cell-Free mMIMO Systems Under Channel Aging," in IEEE Communications Letters, vol. 25, no. 7, pp. 2206-2210, July 2021, doi: 10.1109/LCOMM.2021.3073778.

23. M. Shalaby, H. M. Hussein, and M. Shokair, "The cell-free mMIMO networks: mathematical analysis and performance evaluation," Telecommunication Systems, Springer, 77, 625-641 (2021). Available on this link: https://doi.org/10.1007/s11235-021-00776-z. 


\section{Supplementary Files}

This is a list of supplementary files associated with this preprint. Click to download.

- Highlights.docx 